\title{
Tiling a Rectangle with Polyominoes
}

\author{
Olivier Bodini ${ }^{1}$ \\ ${ }^{1}$ LIRMM, 161, rue ADA, 34392 Montpellier Cedex 5, France
}

\begin{abstract}
A polycube in dimension $d$ is a finite union of unit $d$-cubes whose vertices are on knots of the lattice $\mathbb{Z}^{d}$. We show that, for each family of polycubes $E$, there exists a finite set $F$ of bricks (parallelepiped rectangles) such that the bricks which can be tiled by $E$ are exactly the bricks which can be tiled by $F$. Consequently, if we know the set $F$, then we have an algorithm to decide in polynomial time if a brick is tilable or not by the tiles of $E$.

please also repeat in the submission form
\end{abstract}

Keywords: Tiling, Polyomino

\section{Introduction}

A polycube in dimension d (or more simply a polycube) is a fi nite -not necessarily connected- union of unit cubes whose vertices are on nodes of the lattice $\mathbb{Z}^{d}$. Given a family (fi nite or not) of polycubes $E$, a polycube $P$ is tilable by $E$ if there exists a fi nite sub-family $E_{1}$ of $E$ such that $P$ is a union of translated copies of disjoint interiors of elements of $E_{1}$. An important problem about polyominoes tiling (polycubes in dimension 2) concerns their capacity to tile some rectangles (see works of Klarner [7], Golomb [5,6], Reid [9], Marshall [8], Dahlke [4],...). Especially, Klarner has defi ned the notion of polyomino order as the smallest number of congruent copies of a polyomino which form a rectangle. The question whether there exists a polyomino of odd order $n \geq 5$ is totally open and still remains one of the great discoveries to be made on the polyominoes theory. To understand the diffi culty of that problem, we can remind ourselves that the order (seen as a function) is not algorithmically calculable [1]. The main subject of this paper is to show that, for each family of polycubes $E$, there exists a fi nite set $F$ of bricks (parallelepiped rectangles) such that the bricks that can be tiled by $E$ are exactly the bricks that can be tiled by $F$. In other words, the set of bricks which can be tiled by a fi xed family of polycubes is generated by a fi nite set of bricks called the prime bricks. The proof of this theorem cannot be constructive. In fact, given a family $E$ of polycubes, the problem whether "there exists a rectangle which can be tiled by $E$ " is algorithmically undecidable [1]. Thus, a theorem of fi nite basis type is a bit surprising, although the determination of the cardinality of this basis remains undecidable.

\section{Algebraic Relaxation}

We are going to deal with algebraically simpler objects : for that, we put weights on the cubes of the polycube (we identify a polycube by the set of cubes which compose it). This brings us to introduce the following formal defi nition : Let $\mathbb{A}$ be a unitary ring, an $\mathbb{A}$-weighted polycube is a map with fi nite support 
$P$ from the set of cubes of the lattice $\mathbb{Z}^{d}$ into the ring $\mathbb{A}$. For each cube $c$, the number $P(c)$ is called the weight of $P$ in $c$. The space $P_{\mathbb{A}}$ of the $\mathbb{A}$-weighted polycubes has a natural structure of free $\mathbb{A}$-module and cubes of weight 1 clearly form a basis of $P_{\mathbb{A}}$. Thus, we have a canonical injection of the set of polycubes in the $\mathbb{A}$-module of $\mathbb{A}$-weighted polycubes. We can henceforth introduce the notion of $\mathbb{A}$-tiling in the following way : An $\mathbb{A}$-weighted polycube $P$ will be said $\mathbb{A}$-tilable by a family of $\mathbb{A}$-weighted polycubes (called the tiles) if and only if $P$ is an $\mathbb{A}$-linear combination of translated copies of elements of this family. Consequently, if a polycube $P$ is tilable by a family of tiles $E$, then $P$ is necessarily $\mathbb{A}$-tilable by the family $E$. This provides interesting necessary conditions for the tilability by a family of tiles for a polycube $P$ (see [3] for a development of this idea).

We naturally identify the unit cube $\left(a_{1}, a_{2}, \ldots, a_{d}\right)+[0,1]^{d}$ of $\mathbb{R}^{d}$ to the vector $\left(a_{1}, a_{2}, \ldots, a_{d}\right)$. Moreover, for $a=\left(a_{1}, \ldots, a_{d}\right) \in \mathbb{Z}^{d}$, we put by convention $X^{a}=X_{1}^{\frac{a_{1}+\left|a_{1}\right|}{2}} \ldots X_{d}^{\frac{a_{d}+\left|a_{d}\right|}{2}} Y_{1}^{\frac{\left|a_{1}\right|-a_{1}}{2}} \ldots Y_{d}^{\frac{\left|a_{d}\right|-a_{d}}{2}}$ (this does not constitute all the monomials in $X_{1}, \ldots, X_{d}, Y_{1}, \ldots, Y_{d}$ but only monomials which have not simultaneously some $X_{i}$ and some $Y_{i}$ ).

For each $\mathbb{A}$-weighted polycube $P$, the polynomial :

$$
Q_{P}=\sum_{\left(a_{1}, \ldots, a_{d}\right) \in \mathbb{Z}^{d}} P\left(\left(a_{1}, \ldots, a_{d}\right)\right) X^{\left(a_{1}, \ldots, a_{d}\right)} \text { of } \mathbb{A}\left[X_{1}, \ldots, X_{d}, Y_{1}, \ldots, Y_{d}\right] \text { is the polynomial of } P .
$$

For example, the polycube $P$ in dimension 2 made of the unit squares $(0,1),(0,0),(1,0),(2,0)$ has for polynomial $Q_{P}=X_{2}+1+X_{1}+X_{1}^{2}$. Similarly, the polycube $b=\left\{\left(x_{1}, \ldots, x_{d}\right) \in \mathbb{R}^{d}\right.$ such as $\left.0 \leq x_{i} \leq b_{i}\right\}$ is called the brick of size $b_{1}, \ldots, b_{d}$ and denoted by $b_{1} \times \ldots \times b_{d}$. Its polynomial is $Q_{b}=\prod_{i=1}^{d} \frac{\left(X_{i}^{b_{i}}-1\right)}{X_{i}-1}$.

Lemma 2.1 The space $P_{\mathbb{A}}$ is isomorphic to $\mathbb{A}\left[X_{1}, \ldots, X_{d}, Y_{1}, \ldots, Y_{d}\right] /\left\langle\left(X_{1} Y_{1}-1\right), \ldots,\left(X_{d} Y_{d}-1\right)\right\rangle_{\mathbb{A}}$ where $\left\langle\left(X_{1} Y_{1}-1\right), \ldots,\left(X_{d} Y_{d}-1\right)\right\rangle_{\mathbb{A}}$ denotes the ideal of $\mathbb{A}\left[X_{1}, \ldots, X_{d}, Y_{1}, \ldots, Y_{d}\right]$ generated by

$$
\left(X_{1} Y_{1}-1\right), \ldots,\left(X_{d} Y_{d}-1\right) \text {. }
$$

Proof. There exists a unique linear map $f$ from $\mathbb{A}\left[X_{1}, \ldots, X_{d}, Y_{1}, \ldots, Y_{d}\right]$ to $P_{\mathbb{A}}$ such that $f\left(X_{1}^{a_{1}} Y_{1}^{b_{1}} \ldots X_{d}^{a_{d}} Y_{d}^{b_{d}}\right)$ is the unit cube $\left(a_{1}-b_{1}, \ldots, a_{d}-b_{d}\right)$ of weight 1 (since monomials form a basis of $\mathbb{A}\left[X_{1}, \ldots, X_{d}, Y_{1}, \ldots, Y_{d}\right]$ ).

$f$ is surjective because, for each $\mathbb{A}$-weighted polycube $P$, we have $f\left(Q_{P}\right)=P$. We can conclude by proving that $\operatorname{ker}(f)=\left\langle\left(X_{1} Y_{1}-1\right), \ldots,\left(X_{d} Y_{d}-1\right)\right\rangle_{\mathbb{A}}$. As we can write (in proceeding by successive divisions by $\left.\left(X_{1} Y_{1}-1\right), \ldots,\left(X_{d} Y_{d}-1\right)\right)$ every polynomial $Q$ in the form $Q=R+\sum_{i=1}^{d} Q_{i}\left(X_{i} Y_{i}-1\right)$ where $R$ belongs to the $\mathbb{A}$-module generated by the family $\left\{X^{a} ; a \in \mathbb{Z}^{d}\right\}$, we see that $f(Q)$ is the empty polycube, denoted by 0 (it is the polycube $P$ of weight 0 on all the cubes in the space) if and only if $f(R)=0$ (because $f\left(Q_{i}\left(X_{i} Y_{i}-1\right)\right)=0$ by linearity and by defi nition of $\left.f\right)$. Moreover, it is clear that $f(R)=0 \Leftrightarrow R=0$ and that $R=0 \Leftrightarrow Q \in\left\langle\left(X_{1} Y_{1}-1\right), \ldots,\left(X_{d} Y_{d}-1\right)\right\rangle_{\mathbb{A}}$, so $\operatorname{ker}(f)=\left\langle\left(X_{1} Y_{1}-1\right), \ldots,\left(X_{d} Y_{d}-1\right)\right\rangle_{\mathbb{A}}$.

We denote by $L$ the linear map from $P_{\mathbb{A}}$ into $\mathbb{A}\left[X_{1}, \ldots, X_{d}, Y_{1}, \ldots, Y_{d}\right] /\left\langle\left(X_{1} Y_{1}-1\right), \ldots,\left(X_{d} Y_{d}-1\right)\right\rangle_{\mathbb{A}}$ which associates to $P$ the polynomial $Q_{P}$ and by $L^{\prime}$ the linear quotient map : $f /\left\langle\left(X_{1} Y_{1}-1\right), \ldots,\left(X_{d} Y_{d}-1\right)\right\rangle_{\mathbb{A}}$ from $\mathbb{A}\left[X_{1}, \ldots, X_{d}, Y_{1}, \ldots, Y_{d}\right] /\left\langle\left(X_{1} Y_{1}-1\right), \ldots,\left(X_{d} Y_{d}-1\right)\right\rangle_{\mathbb{A}}$ into $P_{\mathbb{A}}$.

We can notice immediately that $L$ and $L^{\prime}$ are reciprocal maps.

Lemma 2.2 Let $\mathbb{A}$ be a unitary ring and $E$ a set of $\mathbb{A}$-weighted polycubes. An $\mathbb{A}$-weighted polycube $P$ is $\mathbb{A}$-tilable by $E$ if and only if $Q_{P} \in\left\langle Q_{P^{\prime}} \text { with } P^{\prime} \in E, X_{1} Y_{1}-1, \ldots, X_{d} Y_{d}-1\right\rangle_{\mathbb{A}}$. 
Proof. By defi nition, an $\mathbb{A}$-weighted polycube $P$ is $\mathbb{A}$-tilable by $E$ if and only if there exists for all $1 \leq i \leq t$, $\lambda_{i} \in \mathbb{A}, P^{i} \in E$ and $a^{i}=\left(a_{1}^{i}, \ldots, a_{d}^{i}\right) \in \mathbb{Z}^{d}$ such that $P=\sum_{i=1}^{t} \lambda_{i} P_{\left(a_{1}^{i}, \ldots, a_{d}^{i}\right)}^{i}$ where $P_{\left(a_{1}^{i}, \ldots, a_{d}^{i}\right)}^{i}$ denotes the translated of $P^{i}$ by the vector $\left(a_{1}^{i}, \ldots, a_{d}^{i}\right)$. By the isomorphism $L$, this is equivalent to $Q_{P}=\sum_{i=1}^{t} \lambda_{i} X^{a^{i}} Q_{P^{i}}$ in $\mathbb{A}\left[X_{1}, \ldots, X_{d}, Y_{1}, \ldots, Y_{d}\right] /\left\langle\left(X_{1} Y_{1}-1\right), \ldots,\left(X_{d} Y_{d}-1\right)\right\rangle_{\mathbb{A}}$ and so $P$ is $\mathbb{A}$-tilable by $E$ if and only if $Q_{P} \in$ $\left\langle Q_{P^{\prime}} \text { with } P^{\prime} \in E, X_{1} Y_{1}-1, \ldots, X_{d} Y_{d}-1\right\rangle_{\mathbb{A}}$.

In the following, the set $\left\langle Q_{P^{\prime}} \text { with } P^{\prime} \in E, X_{1} Y_{1}-1, \ldots, X_{d} Y_{d}-1\right\rangle_{\mathbb{A}}$ is denoted by $I(E)$. This is what we call the ideal of $E$.

\section{Existence of Finite Generated Families}

Firstly (until the theorem 3.4), we are going to present a short proof of a fundamental result due to Barnes [2].

In order to do that, we are going to defi ne new notations. A color $c$, is a $d$-tuple $\left(c_{1}, \ldots, c_{d}\right)$ where $c_{i}$ belongs to $\mathbb{N} \backslash\{1\}$. We say that a color $\left(c_{1}, \ldots, c_{n}\right)$ conceals a brick $B_{1} \times \ldots \times B_{d}$ if there exists an $i$ such that $c_{i}$ divides $B_{i}$.

Let $c$ be a color, we denote by $e^{i c}$ the $d$-tuple $\left(x_{1}, \ldots, x_{d}\right)$ where $x_{i}=\left\{\begin{array}{ll}1 & \text { if } c_{i}=0 \\ e^{\frac{2 i \pi}{c_{i}}} & \text { otherwise }\end{array}\right.$.

Lemma 3.1 For every brick $B$ (embedded in $\mathbb{C}$ ) and for every color $c, Q_{B}(\alpha(c))$ is null if and only $c$ conceals $B$.

Proof. $Q_{B}=\prod_{i=1}^{d} \frac{\left(X_{i}^{B_{i}}-1\right)}{X_{i}-1}=0$ if and only if there exists an $i$ such that the factor $\frac{X_{i}^{B_{i}}-1}{X_{i}-1}$ in $Q_{B}$ vanishes during the evaluation. Thus, $c_{i} \mid B_{i}$.

Let $B$ be a brick $B_{1} \times \ldots \times B_{d}$ and let $\left\{b_{1}^{1} \times \ldots \times b_{d}^{1}, \ldots, b_{1}^{m} \times \ldots \times b_{d}^{m}\right\}$ be a set $E$ of bricks. We denote by $m_{i}$ the least common multiple of $b_{i}^{1}, \ldots, b_{i}^{m}$. A color $\left(c_{1}, \ldots, c_{d}\right)$ is $\left(m_{1}, \ldots, m_{d}\right)$-primal if every positive $c_{i}$ is a prime power which divides $m_{i}$. The set $C_{E}$ will be the set of the $\left(m_{1}, \ldots, m_{d}\right)$-primal colors which conceal all bricks in $E$.

Lemma 3.2 If a brick $B$ is $\mathbb{C}$-tilable by $E$, then $\forall c \in C_{E}$, c conceals $B$.

Proof. It is clear that $Q_{B}=\sum_{b \in E} P_{b} Q_{b}$ where $P_{b}$ is a polynomial. Now, let $c$ be an $\left(m_{1}, \ldots, m_{d}\right)$-primal color which conceals all bricks in $E$, then $\alpha(c)$ is a zero of every $Q_{b}$. So, $\alpha(c)$ is a zero of $Q_{B}$ and by lemma 3.1, $d$ conceals $B$.

In the sequel, we denote by $v_{p}(n)$ the $p$-valuation of $n$, that is to say the greatest integer $k$ such that $p^{k} \mid n$.

Lemma 3.3 Let $\left\{b_{1}^{1} \times \ldots \times b_{d}^{1}, \ldots, b_{1}^{m} \times \ldots \times b_{d}^{m}\right\}$ be a set $E$ of bricks and $B_{1} \times \ldots \times B_{d}$ a brick $B$. If the brick $B$ verifies the following conditions :

1. $\forall c \in C_{E}, c$ conceals $B$.

2. $\forall i$, there exists a prime number $p_{i}$ such that for every prime number $p \neq p_{i}$, we get $v_{p}\left(B_{i}\right) \geq$ $\max _{k}\left(v_{p}\left(b_{i}^{k}\right)\right)$ 
Then $B_{1} \times \ldots \times B_{d}$ is a multiple of one of the bricks of $E$.

Proof. Let $c=\left(c_{1}, \ldots c_{d}\right)$ be the color where $c_{i}=p_{i}^{v_{p_{i}}\left(B_{i}\right)+1}$. By lemma 3.1, it follows that $Q_{B}(\alpha(c)) \neq 0$. So, there exists a brick $b$ in $E$ such that the evaluation of $Q_{b}$ in $\alpha(c)$ is not null too (this follows from the fi rst condition of the lemma). So, for every $i, c_{i}$ does not divide $b_{i}$, which implies $v_{p_{i}}\left(B_{i}\right)+1>v_{p_{i}}\left(b_{i}\right)$. Now, by hypothesis, for every $p \neq p_{i}, v_{p}\left(B_{i}\right) \geq v_{p}\left(b_{i}\right)$. We conclude that for every $i, b_{i}$ divides $B_{i}$.

We note $\mathbb{B}_{>k}$ the set of bricks $B$ such that all the edges of $B$ are greater than $k$. In other words, $\mathbb{B}_{>k}=$ $\left\{b_{1} \times \ldots \times b_{d}\right.$ with $\left.b_{i}>k\right\}$. Now, we can prove the following theorem due to Barnes [2]:

Theorem 3.4 Let $E$ be a finite set of bricks, then there exists an integer $k$, which depends uniquely on this set, such that for each brick $B \in \mathbb{B}_{>k}, B$ is tilable by $E$ if and only if $B$ is $\mathbb{C}$-tilable by $E$.

Proof. Let $\left\{b_{1}^{1} \times \ldots \times b_{d}^{1}, \ldots, b_{1}^{m} \times \ldots \times b_{d}^{m}\right\}$ be the bricks in $E$. We recall that $m_{i}$ is the least common multiple of $b_{i}^{1}, \ldots, b_{i}^{m} . m_{i}$ can be factorized in $m_{i}=q_{i, 1} \ldots q_{i, t_{i}}$ where each $q_{i, j}$ is a prime power and $q_{i, j} \wedge q_{i, j^{\prime}}=1$ for $j \neq j^{\prime}$. Suppose that $B$ is $\mathbb{C}$-tilable by $E$. By lemma 3.2, $\forall c \in C_{E}, c$ conceals $B$. Let $\delta_{i}$ be the greatest common divisor of $m_{i}$ and $B_{i}$. Now, we can find $u_{i, j}$ such that for every $i$ :

$$
\frac{m_{i}}{q_{i, 1}} u_{i, 1} \delta_{i}+\ldots+\frac{m_{i}}{q_{i, t_{i}}} u_{i, t_{i}} \delta_{i}=B_{i}
$$

If $B_{i}$ is suffi ciently large, all the $u_{i, j}$ can be chosen non-negative and then the non-empty bricks $\frac{m_{1}}{q_{1, j_{1}}} u_{1, j_{1}} \delta_{1} \times$ $\ldots \times \frac{m_{d}}{q_{d, j_{d}}} u_{d, j_{d}} \delta_{d}$ with $\left(j_{1}, \ldots, j_{d}\right)$ belonging to $\left\{1, \ldots, t_{1}\right\} \times \ldots \times\left\{1, \ldots, t_{d}\right\}$ constitutes a tiling of $B_{1} \times \ldots \times$ $B_{d}$. Then $\forall c \in C_{E}, c$ conceals all this bricks. Indeed, $c_{i} \mid m_{i}$ by defi nition of the $\left(m_{1}, \ldots, m_{d}\right)$-primal colors and as $c$ conceals $B$, there exists an $i$ such that $c_{i} \mid B_{i}$. So, for a $j \in\left\{1, \ldots, t_{i}\right\}$, we get $c_{i} \mid \delta_{i} \frac{m_{1}}{q_{1, j_{1}}} u_{1, j_{1}}$ and thus, $c$ conceals the non-empty bricks $\frac{m_{1}}{q_{1, j_{1}}} u_{1, j_{1}} \delta_{1} \times \ldots \times \frac{m_{d}}{q_{d, j_{d}}} u_{d, j_{d}} \delta_{d}$. Now, this bricks verify the conditions of the lemma 3.3. In order to prove that, we can observe that if $p$ doesn't divide $q_{i, j_{i}}$, then $v_{p}\left(\frac{m_{i}}{q_{i, j_{i}}} u_{i, j_{i}} \delta_{i}\right) \geq v_{p}\left(m_{i}\right)=\max _{k}\left(v_{p}\left(b_{i}^{k}\right)\right)$. So, each of this bricks are multiple of one of the bricks of $E$ and we have find a tiling of $B$.

Using a trivial bound of the Frobenius' problem, we can remark that $k \leq \max \left(m_{i}^{2}\right)$.

We write $[E]_{\mathbb{A}}$ the set of all bricks which are $\mathbb{A}$-tilable by $E$ and $[E]$ the set of all bricks which are tilable (in classical sense) by $E$.

Lemma 3.5 Let $\mathbb{A}$ be a notherian ring and $E$ a set of $\mathbb{A}$-weighted polycubes, then there exists a finite subset $F$ of $E$ such that $[E]_{\mathbb{A}}=[F]_{\mathbb{A}}$.

Proof. $P \in[E]_{\mathbb{A}}$ if and only if $Q_{P} \in\left\langle Q_{P^{\prime}} \text { with } P^{\prime} \in E, X_{1} Y_{1}-1, \ldots, X_{d} Y_{d}-1\right\rangle_{\mathbb{A}}$, but as $\mathbb{A}\left[X_{1}, \ldots, X_{d}, Y_{1}, \ldots, Y_{d}\right]$ is a nœtherian ring, there exists a fi nite subset $F$ of $E$ such that $\left\{Q_{P^{\prime}}\right.$ with $\left.P^{\prime} \in F, X_{1} Y_{1}-1, \ldots, X_{d} Y_{d}-1\right\}$ is a generating family of $\left\langle Q_{P^{\prime}} \text { with } P^{\prime} \in E, X_{1} Y_{1}-1, \ldots, X_{d} Y_{d}-1\right\rangle_{\mathbb{A}}$. Otherwise, for each integer $n$ and $P_{1}, \ldots, P_{n}$ polycubes of $E$, there exists a polycube $P_{n+1}$ in $E$ such that

$$
Q_{P_{n+1}} \notin\left\langle Q_{P_{1}}, \ldots, Q_{P_{n}}, X_{1} Y_{1}-1, \ldots, X_{d} Y_{d}-1\right\rangle_{\mathbb{A}}
$$

and so, we can obtain a non stationary increasing sequence of ideals.

Let $E, E^{\prime}$ and $C$ be some families of polycubes, $E$ and $E^{\prime}$ are two paddles (resp. $\mathbb{A}$-paddles where $\mathbb{A}$ is a unitary ring) equivalent on $C$ if and only if $\forall P \in C$, the following statements are logically equivalent :

- $P$ is tilable (resp. $\mathbb{A}$-tilable) by $E$, 
- $P$ is tilable (resp. $\mathbb{A}$-tilable) by $E^{\prime}$.

We can now prove the main theorem for the $\mathbb{A}$-tiling. More explicitly :

Corollary 3.6 Let $\mathbb{A}$ be a nætherian ring and $E$ a set of $\mathbb{A}$-weighted polycubes, then there exists a finite set $F$ of bricks such that $E$ et $F$ are equivalent $\mathbb{A}$-paddles for the bricks.

Proof. We have clearly $\left[[E]_{\mathbb{A}}\right]_{\mathbb{A}}=[E]_{\mathbb{A}}$ and with the lemma 3.5 applied to $[E]_{\mathbb{A}}$, there exists $F$ such that $[F]_{\mathbb{A}}=\left[[E]_{\mathbb{A}}\right]_{\mathbb{A}}=[E]_{\mathbb{A}}$.

Lemma 3.7 Let $E$ be a set of bricks, then there exists a finite subset $F$ of $E$ such that $[E]=[F]$.

Proof. By the lemma 3.5 there exists a fi nite subset $E_{1}$ of $E$ such that $[E]_{\mathbb{C}}=\left[E_{1}\right]_{\mathbb{C}}$.

Moreover, by the theorem 3.4, there exists $k$ such that $\left[E_{1}\right]_{\mathbb{C}} \cap \mathbb{B}_{>k}=\left[E_{1}\right] \cap \mathbb{B}_{>k}$. So, we have :

$$
\left[E_{1}\right]_{\mathbb{C}} \cap \mathbb{B}_{>k}=\left[E_{1}\right] \cap \mathbb{B}_{>k} \subset[E] \cap \mathbb{B}_{>k} \subset[E]_{\mathbb{C}} \cap \mathbb{B}_{>k}=\left[E_{1}\right]_{\mathbb{C}} \cap \mathbb{B}_{>k}
$$

This involves that $\left[E_{1}\right] \cap \mathbb{B}_{>k}=[E] \cap \mathbb{B}_{>k}$

The elements of $[E]$ which are not in $\left[E_{1}\right]$ are bricks such that the length of at least one of their edges is less than $k$.

For $1 \leq q \leq k$ and $1 \leq r \leq d$, we put $B_{q, r}$ the set of bricks of [E] such that the length of the $r$-th edge is $q$.

We conclude by showing that there exists a fi nite family of polycubes $E_{2}$ such that $[E]=\left[E_{1} \cup E_{2}\right]$. We are going to proceed by recurrence on $d$ (the dimension of the space).

If $d=1$, for each $q$, the set $B_{q, 1}$ is constituted by at most one brick and so $\left[E_{1} \cup \underset{1 \leq q \leq k}{\cup} B_{q, 1}\right]=[E]$.

Suppose the lemma verifi ed for rank $d-1$. We put :

$$
\begin{array}{r}
C_{q, r}=\left\{a_{1} \times \ldots \times a_{r-1} \times a_{r+1} \times \ldots \times a_{d}\right. \text { such that } \\
\left.a_{1} \times \ldots \times a_{r-1} \times q \times a_{r+1} \times \ldots \times a_{d} \in B_{q, r}\right\}
\end{array}
$$

So $C_{q, r}$ is a set of bricks of dimension $d-1$ obtained by canonical projection of $B_{q, r}$. By recurrence hypothesis, there exists $D_{q, r}$ a fi nite set of bricks such that $\left[D_{q, r}\right]=\left[C_{q, r}\right]$.

Let

$$
\begin{array}{r}
E_{q, r}=\left\{a_{1} \times \ldots \times a_{r-1} \times q \times a_{r+1} \times \ldots \times a_{d}\right. \text { such that } \\
\left.a_{1} \times \ldots \times a_{r-1} \times a_{r+1} \times \ldots \times a_{d} \in D_{q, r}\right\},
\end{array}
$$

then $E_{q, r}$ is fi nite and we clearly have $\left[E_{q, r}\right]=\left[B_{q, r}\right]$. So $E_{1} \cup \underset{\substack{1 \leq q \leq k \\ 1 \leq r \leq d}}{\cup} E_{q, r}$ is fi nite and $\left[E_{1} \cup \underset{\substack{1 \leq q \leq k \\ 1 \leq r \leq d}}{\cup} E_{q, r}\right]=[E]$.

Remark 3.8 The lemma 3.4 works on the same principle as the lemma of Dickson which allows us to obtain the notheriality of

$$
\mathbb{C}\left[X_{1}, \ldots, X_{n}\right] .
$$

Theorem 3.9 Let $E$ be a set of polycubes, then there exists a finite set $F$ of bricks, such that $E$ and $F$ are equivalent paddles on the bricks. 
Proof. We have clearly $[[E]]=[E]$ and $[E]$ is a set of bricks, so by the lemma 3.4 , there exists a fi nite subset $F$ of $[E]$ such that $[F]=[[E]]=[E]$.

We can observe, in corollary, that for every set $E$ of polycubes, if we make the hypothesis that we know a fi nite family $F$ of bricks which is an equivalent paddle, we can determine rapidly if a given brick $B$ is tilable by $E$. Indeed, there are two possibilities :

- Brick $B$ has all its edges of length greater than $k$ ( $k$ is supposed determined) and then it suffi ces to verify that $B$ is $\mathbb{C}$-tilable by $E$ (we can do this in polynomial time using generalized division algorithm [3])

- $B$ is an element of the fi nite set $F$.

\section{Rectangles which can be Tiled by Small Polyominoes}

We propose in this section a survey of the results known on the tilable rectangles by congruent copies of a given edge-connected polyomino. (We cover only the cases where the number of squares of the polyomino is less than 7. The unmentioned polyominoes with less than 7 squares cannot tile any rectangles). Some of this results deserve a citation, the other one are unpublished results and they can be easily verifi ed. We can observe that for some polyominoes the number of rectangles is huge, they are no explanation for this fact. For each polyomino, we present only an "half-base" of rectangles. To obtain a generating base, we have to symmetrize this half-base by adding $b \times a$ if $a \times b$ is in the half-base. 


\begin{tabular}{|c|c|c|c|c|c|}
\hline$\square$ & $1 \times 1$ & & \multirow{3}{*}{$\begin{array}{l}\square \amalg D \\
\boxplus \square\end{array}$} & \multirow{3}{*}{$\begin{array}{l}1 \times 6 \\
2 \times 3\end{array}$} & \\
\hline$\square$ & $1 \times 2$ & & & & \\
\hline पा & $1 \times 3$ & & & & \\
\hline 廿 & $2 \times 3,5 \times 9$ & [7] & एाम & $\begin{array}{l}2 \times 6,7 \times 12,9 \times\{14,16,34\} \\
10 \times 15,11 \times 18,15 \times 15\end{array}$ & [9] \\
\hline$\square \square$ & $1 \times 4$ & & 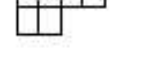 & $6 \times 11.9 \times 10$ & \\
\hline$Q_{0}$ & $2 \times 4,3 \times 8$ & [7] & प & $4 \times 6,5 \times 12$ & \\
\hline$\square$ & $4 \times 4$ & [10] & $\uplus \square$ & $3 \times 4$ & \\
\hline$\boxplus$ & $2 \times 2$ & & $\boxplus$ & $3 \times 4$ & \\
\hline$\square 11$ & $1 \times 5$ & & $\bigoplus$ & $9 \times\{12,20,28\}, 12 \times 13$ & [7] \\
\hline प1口 & $2 \times 5,7 \times 15$ & [9] & 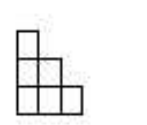 & $3 \times 4$ & \\
\hline$\square \square$ & See note $n^{\circ} 1$ & & पण & See note $n 2$ & \\
\hline$\bigoplus$ & $2 \times 5,7 \times 15$ & [7] & & & \\
\hline
\end{tabular}

Fig. 1: Rectangles which can be Tiled by Small Polyominoes Rectangles which can be Tiled by Small Polyominoes. 
Note $\mathrm{n}^{\circ} 1$ :

$5 \times 10$

$9 \times\{20,30,45,55\}$

$10 \times\{14,16,23,27\}$

$11 \times\{20,30,35,45\}$

$12 \times\{50,55,60,65,70,75,80,85,90,95\}$

$13 \times\{20,30,35,45\}$

$14 \times 15$

$15 \times\{15,16,17,19,21,22,23\}$

$17 \times\{20,25\}$

$18 \times\{25,35\}, 22 \times 25$

Note ${ }^{\circ} 2$ :

$23 \times 24$

$24 \times\{29,35,41,47,53,59,63,65,71,77,83,89,95,101,102,103,107,108,113,114,119,120 \ldots\}$

This polyomino is the hexomino $Y$. It is the smallest case which is still open.

\section{Acknowledgements}

The author would like to thank M. Reid for his helpful e-mail about the board in section 4.

\section{References}

[1] R. Berger, The undecidability of the domino problem, Mem. Amer. Math. Soc. 66 (1966).

[2] F.W. Barnes, Algebraic theory of brick packing, Discrete Math. 42 (1982) 129-144.

[3] O. Bodini, Z-tilings of polyominoes and standard basis, to appear in FPSAC'02 proceeding.

[4] K.A. Dahlke, The Y-hexomino has order 92, J.C.T. Series A 51 (1989) 125-126.

[5] S. W. Golomb, Tiling with polyominoes, J.C.T. Series A 1 (1966) 280-296.

[6] S. W. Golomb, Polyominoes which tile rectangles, J.C.T. Series A 51 (1989) 117-124.

[7] D.A. Klarner, Packing a rectangle with congruent $n$-ominoes, J.C.T. Series A 7 (1969) 107-115.

[8] W.R. Marshall, Packing rectangles with congruent polyominoes, J.C.T. Series A 77 (1997) 181-192.

[9] M. Reid, Tiling rectangles and half strips with congruent polyominoes, J.C.T. Series A 80 (1997) 106-123.

[10] D.W. Walkup, Covering a rectangle with T-tetraminoes, Amer. Math. Monthly 72 (1965), 986-988. 